\title{
Article \\ New Insights on Hemopexin Binding to Hemin and Hemoglobin
}

\author{
Guilherme C. Lechuga ${ }^{1,2}$ Paloma Napoleão-Pêgo ${ }^{1,2}$, Carlos M. Morel ${ }^{1}$, Salvatore G. De-Simone1, ${ }^{1,2,3,}$
}

${ }^{1}$ FIOCRUZ, Center for Technological Development in Health (CDTS)/National Institute of Science and Technology for Innovation on Neglected Population Diseases (INCT-IDPN), Rio de Janeiro, RJ, Brazil, 21040-900; guilherme.lechuga@cdts.fiocruz.br (G.C.L.); napoleaopego@ioc.fiocruz.br (P.N.P.); carlos.morel@cdts.fiocruz.br (C.M.M.); salvatore.simone.fiocruz.br (S.G.D-S.)

${ }^{2}$ Laboratory of Epidemiology and Molecular Systematics (LESM), Oswaldo Cruz Institute, FIOCRUZ, Rio de Janeiro 21040-900, RJ, Brazil.

${ }^{3}$ Federal Fluminense University, Biology Institute, Department of Cellular and Molecular Biology, Niterói, 24020141, RJ, Brazil

* Correspondence: salvatore.simone@fiocruz.br; Tel.: +55-213-865-8183

\begin{abstract}
Background: Hemopexin (Hx) is a plasma glycoprotein that scavenges heme (Fe(III) protoporphyrin IX), Hx have important implication in hemolytic disorders and hemorrhagic condition because the release of hemoglobin increase labile heme, which is potentially toxic producing oxidative stress. Hx has been considered for therapeutic use and diagnostics. In this work, we analyzed and mapped interaction sequences of Hx with hemin and hemoglobin (2) Methods: Spot-synthesis technique was used to map human hemopexin (P02790) binding to hemin and human hemoglobin, a library of 15 amino acid peptides with a 10-amino acid overlap was designed to represent the entire coding region (aa 1-462) of hemopexin and synthesized onto cellulose membranes. In silico approach was performed to analyze amino acid frequency in identified interaction regions, and molecular docking was applied for protein-protein interaction (3) Results: Seven linear peptide sequences in $\mathrm{Hx}$ were identified to bind hemin ( $\mathrm{H} 1-\mathrm{H} 7)$, and five were described for $\mathrm{Hb}(\mathrm{Hb} 1-\mathrm{Hb} 5)$ interaction, with just two sequences shared between hemin and $\mathrm{Hb}$. Amino acid composition of identified sequences demonstrated that Histidine residues are relevant for heme binding, H105, H293, H373, $\mathrm{H} 400, \mathrm{H} 429$, and $\mathrm{H} 462$ was distributed in $\mathrm{H} 1-\mathrm{H} 7$ peptide sequences, but other residues may also play an important role. Molecular docking analysis demonstrated $\mathrm{Hx}$ association with the $\beta$-chain of $\mathrm{Hb}$, with several hot spot amino acids that coordinated interaction. (4) Conclusions: This study highlights new insights on Hx-hemin binding motifs and protein-protein interactions with $\mathrm{Hb}$. Binding sequences and identified specific peptides can be used for therapeutic purposes and diagnostics, as hemopexin is under investigation to treat different diseases, and there is an urgent need for diagnostics of labile heme for monitoring hemolysis.
\end{abstract}

Keywords: Hemopexin; Hemoglobin; protein-protein binding; hemin

\section{Introduction}

Hemopexin is a plasma glycoprotein that scavenges heme with high affinity [1]. It is a pivotal protein in hemolytic conditions and cell injury in malaria, sickle cell disease (SCD), thalassemia, hemorrhage, and hemorrhagic stroke [2]. The detrimental effect of releasing hemoglobin $(\mathrm{Hb})$ during hemolysis is prevented by the scavenger serum protein haptoglobin that transports $\mathrm{Hb}$ to hepatocytes and macrophages for its degradation [3]. Hemoglobin oxidation releases labile heme (Fe(III) protoporphyrin IX), a source of iron, that potentially produces oxidative stress through the Fenton reaction [4]. However, the exhaustion of $\mathrm{Hx}$ and $\mathrm{Hp}$ binding capacity can lead to oxidative damage that ultimately can induce severe complications, including stroke, thrombosis, and pulmonary embolism [4-6]. Increasing evidence demonstrates the importance of $\mathrm{Hx}$, and knockout mice showed neuronal degeneration and cognitive impairment after induction of intracerebral hemorrhage [7]. Also, Hx prevents heme-induced proinflammatory switching of macrophages toward 
an M1 phenotype [8]. Recently, a heme and iron dysmetabolism was observed in Alzheimer's disease (AD), with a significant increase of hemoglobin subunit $\beta$ in AD [9].

The crystallographic studies showing the three-dimensional structure of the rabbit hemopexin-heme complex revealed that $\mathrm{Hx}$ has 4 -bladed $\beta$-propeller folding motifs similar in the N- and C-terminal domains joined together by a flexible structure hinge sequence. Furthermore, heme has binding sites in histidine residues along the flexible linker region and another in the C-terminal domain [10]. However, interactions can occur with other amino acids. Another open question is whether Hx could interact with hemoglobin, the primary source of heme in blood. Additionally, essential functions of the complex Heme-Hx have also been highlighted, like serine protease and immunomodulatory activity [3].

Considering its significant biological implications, it is crucial to understand the interactions of Hx with heme and hemoglobin. Furthermore, identifying the Hx interactome may substantially impact medicine since $\mathrm{Hx}$ has been reported as a biomarker for hemolysis and sepsis [11]. Also, the administration of Hx can improve the clinical outcome of some diseases, e.g., sickle cell disease [12], the utilization of recombinant Hx [13], plasmapheresis, or heme-binding peptides have commercial potential for the treatment of the above-cited pathologies. Here we applied the microarray of peptides and in silico analysis to map the interactions of $\mathrm{Hx}$ with hemin and hemoglobin, seven linear peptide sequences in $\mathrm{Hx}$ were identified to bind hemin, and five were depicted for $\mathrm{Hb}$ interaction.

\section{Results}

\subsection{Identification of the hemopexin-hemin and hemopexin-hemoglobin interaction regions}

To evaluate the interaction regions of $\mathrm{Hx}$ with hemin and $\mathrm{Hb}$, employed the strategy of parallel synthesis of peptides on a cellulose membrane. The Spot Synthesis analysis was used using an array of 15-mer peptides with a ten amino acid overlap representing the $\mathrm{tHx}$ sequence synthesized in situ on a cellulose membrane. Following incubation with hemin/ $\mathrm{Hb}$ and its subsequent detection by hemin peroxidase-like activity and $\mathrm{Hb}$-specific antibodies with corresponding secondary antibodies, several highly reactive spots were revealed that indicated a direct interaction between sequences in $\mathrm{Hx}$ with hemin and $\mathrm{Hb}$ (Figure 1). From the calculated relative intensity percentage, where signal intensities above $50 \%$ were considered the cutoff for a positive reaction, seven individual peptide sequences were defined that interacted with hemin (Figure1A) and five with $\mathrm{Hb}$ (Figure 1B). Secondary structure analysis revealed that most of the interactions sequences in Hx were coiled arranged (7), while few were also in beta-sheet (3). Just one sequence that interacts with $\mathrm{Hb}(\mathrm{Hb} 1)$ was in the alpha-helix structure located in the $\mathrm{N}$-terminal domain (Table 1), but most of the sequences (Hb2-Hb5) are in the coil. Shared only two Hx interaction sequences between hemin and $\mathrm{Hb}$ (Figure 2), peptide fragment RLHIMAGRRL and KSGAQATWTE were present in $\mathrm{H} 4$ and $\mathrm{H} 5$, respectively, and $\mathrm{Hb} 5$. 
A

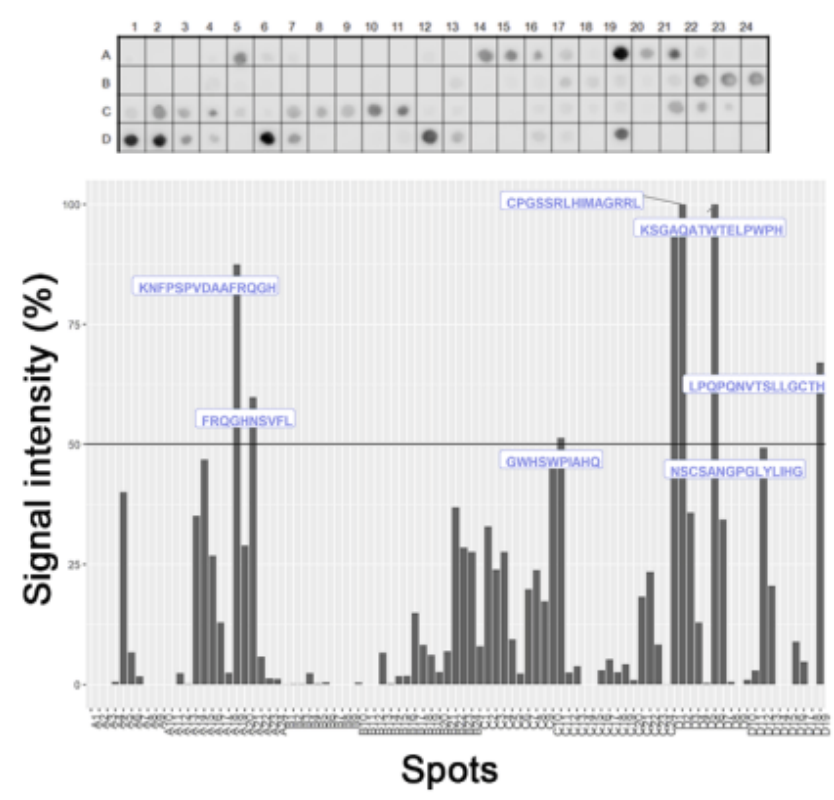

B
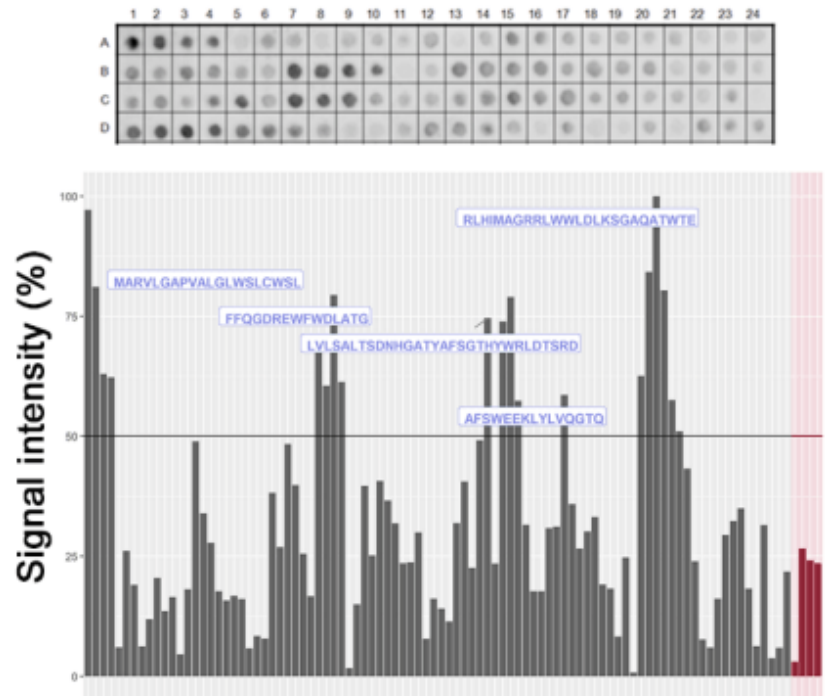

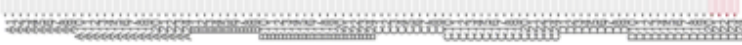

Spots

Figure 1: Linear mapping of hemopexin interaction with hemin and hemoglobin in the SPOT synthesis array (15 residues overlapping 10). (A) The signal intensity of interaction regions of hemopexin sequences with hemin; (B) Signal intensity of hemoglobin binding to hemopexin. Negative control (red bars) represents sequences of $60 \mathrm{kDa}$ chaperonin from Leptospira interrogans (DREKLQERLAKLAG), Toxoplasma gondii - Dense granule protein GRA6 (HPGSVNEFDF), Rabies virus (AVNFPNPPGKGGG), and Cowpox virus (QEVRKYFCV). Each peptide was identified by the Spot-synthesis membrane position numbering (Table S1). Spot intensities below $50 \%$ were considered as background.

Table 1: List of the identified interactions between hemopexin and hemin/hemoglobin and structural properties of these regions.

\begin{tabular}{|c|c|c|c|c|}
\hline Code & Interaction site & Molecule & Position & Secondary structure \\
\hline H1 & KNFPSPVDAAFRQGH & Hemin & 91-105 & $\mathrm{C}+\mathrm{S}$ \\
\hline H2 & FRQGHNSVFL & Hemin & $101-110$ & $\mathrm{~s}$ \\
\hline H3 & GWHSWPIAHQ & Hemin & $291-300$ & C \\
\hline $\mathrm{H} 4$ & CPGSSRLHIMAGRRL & Hemin & $366-380$ & $C+S$ \\
\hline H5 & KSGAQATWTELPWPH & Hemin & $386-400$ & $\mathrm{C}$ \\
\hline H6 & NSCSANGPGLYLIHG & Hemin & $416-430$ & $C+S$ \\
\hline H7 & LPQPQNVTSLLGCTH & Hemin & $448-462$ & $\mathrm{C}$ \\
\hline $\mathrm{Hb} 1$ & $\begin{array}{c}\text { MARVL- } \\
\text { GAPVALGLWSLCWSL }\end{array}$ & Hemoglobin & $1-20$ & $\mathbf{H}$ \\
\hline $\mathrm{Hb} 2$ & FFQGDREWFWDLATG & Hemoglobin & $161-175$ & $\mathrm{C}$ \\
\hline $\mathrm{Hb} 3$ & $\begin{array}{c}\text { LVLSALTS- } \\
\text { DNHGATYAFSGTHY- } \\
\text { WRLDTSRD }\end{array}$ & Hemoglobin & $261-290$ & $\mathrm{C}$ \\
\hline $\mathrm{Hb} 4$ & AFSWEEKLYLVQGTQ & Hemoglobin & $311-324$ & $\mathrm{C}$ \\
\hline Hb5 & $\begin{array}{c}\text { RLHIMAGRRL- } \\
\text { WWLDLKSGAQATWTE }\end{array}$ & Hemoglobin & $371-395$ & C \\
\hline
\end{tabular}



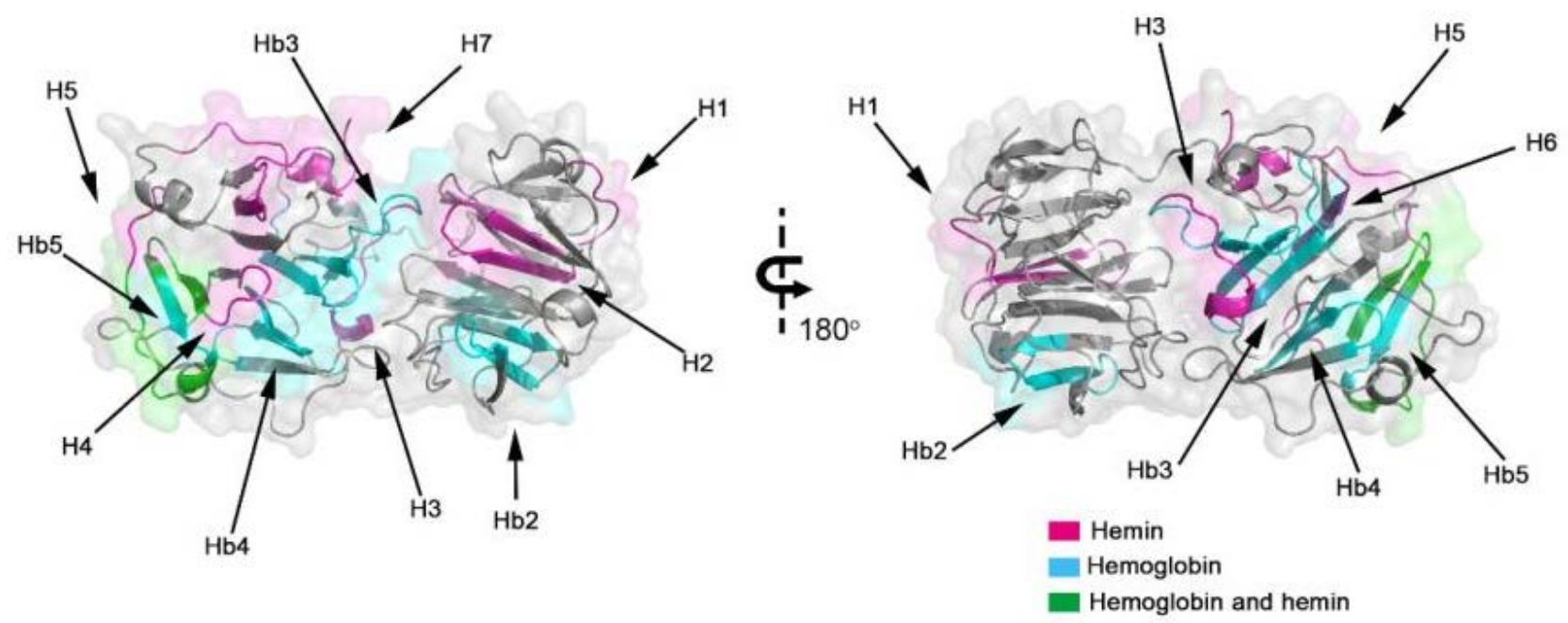

Figure 2: Model of human hemopexin with interaction domains with hemin and hemoglobin. 3D protein conformation and structure were determined using homology model, and interaction regions were highlighted for Hx interaction sites with hemin (magenta), hemoglobin (cyan), and both molecules (green), indicating the position of the 12 linear sequences identified by Spot-synthesis (Table 1). C, coil; S, sheet; H, helix.

\subsection{Frequency of amino acids in hemopexin-hemin/hemoglobin binding sites}

Interaction with heme occurs mainly with histidine residues. The analysis of amino acid frequency in all $\mathrm{Hx}$ peptide sequences showed that leucine ( $\mathrm{L} ; 15,2 \%$ ) was the most frequent amino acid in hemoglobin interaction sites, followed by alanine (A, 10,5\%), tryptophan $(\mathrm{W}, 8,6 \%)$, and glycine $(\mathrm{G}, 8,6 \%)$. Glycine $(\mathrm{G}, 10,5 \%)$ were also frequent amino acids in $\mathrm{Hx} /$ hemin interaction sites, followed by Leucine (L, 9,5\%), Proline (P, 9,5\%), Serine (S, 9,5\%) and Histidine (H, 8,4\%) (Figure 3A). Glycine, histidine, and serine were present in all $\mathrm{Hx}$ interaction sequences with hemin (H1-H7). In contrast, alanine, glycine, leucine, and tryptophan compose $100 \%$ of $\mathrm{Hx} / \mathrm{Hb}$ binding sequences ( $\mathrm{Hb} 1-\mathrm{Hb} 5)$ (Fig.3B). Histidine residue is important in heme-binding proteins. H105, H293, H373, H400, H429, and $\mathrm{H} 462$ were distributed in H1-H7 peptide sequences (Figure 2 and Table 1). 

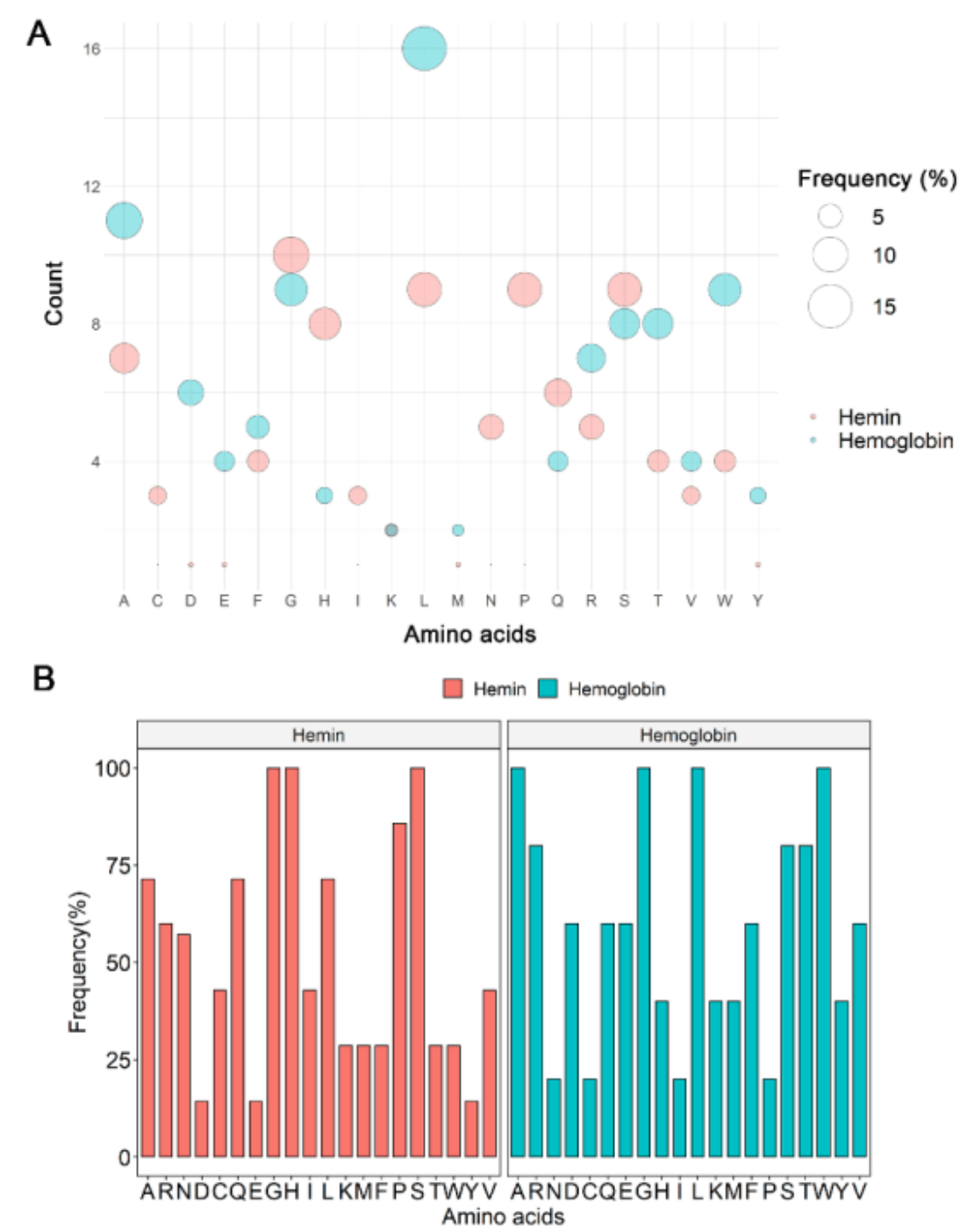

Figure 3: Frequency analysis of the amino acid composition of $\mathrm{Hx}$ interaction sites with hemoglobin and hemin. (A) Global count and frequency of amino acid in $\mathrm{Hx}$ identified sequences. (B) Frequency of amino acid in $\mathrm{Hx}$ interaction sites ( $\mathrm{H} 1-\mathrm{H} 7 \mathrm{and} \mathrm{Hb} 1-\mathrm{Hb} 5)$.

\subsection{Molecular docking analysis of hemopexin and hemoglobin}

This interaction was also be inferred from a molecular docking analysis for protein/protein interactions. The interactome of $\mathrm{Hx}$ was evaluated using network-based analysis with selected highest confidence interactions. Results pointed out that Hx can interact with others serum proteins like albumin (ALB), haptoglobin (HP), and the $\beta$ chain of hemoglobin (HBB). Additionally, human $\mathrm{Hx}$ can interact with fibrinogen gamma chain (FGG), metalloproteinase inhibitor 1 and 2 (TIMP1 and TIMP2), the receptor low-density lipoprotein receptor-related protein 1 (LRP1), and Feline leukemia virus subgroup $C$ receptor-related protein 1 (FLVCR1), a receptor-related with heme export. The possibility of binding the $\mathrm{Hb}$ beta domains was evidenced by a molecular docking assay, showing binding energy of $-528 \mathrm{kcal} / \mathrm{mol}$ (Figure S1). The molecular model indicates a close association between the $\mathrm{Hx}$ and the $\beta$-chain of $\mathrm{Hb}$ (Figure $4 \mathrm{~B}$ and C). Interaction regions showed that five peptide sequences bind strongly to human hemoglobin chains. Hb5 (RLHIMAGRRLWWLDLKSGAQATWTE) had the highest 
reactivity signal in Spot-synthesis, suggesting that this region could contribute to $\mathrm{Hb}-\mathrm{Hx}$ binding. In silico analysis showed that this interaction could be coordinated by four amino acid residues from Hx (His-353; Thr-394; GLU-395 and PRO-397) and two in $\mathrm{Hb}$ (Val-20 and ASP-21) (Figure 4C; Table S2). In addition, hydrogen bonds were evidenced in protein-protein interface and hotspot residues from $\mathrm{Hb} \beta$-chain and $\mathrm{Hx}$.

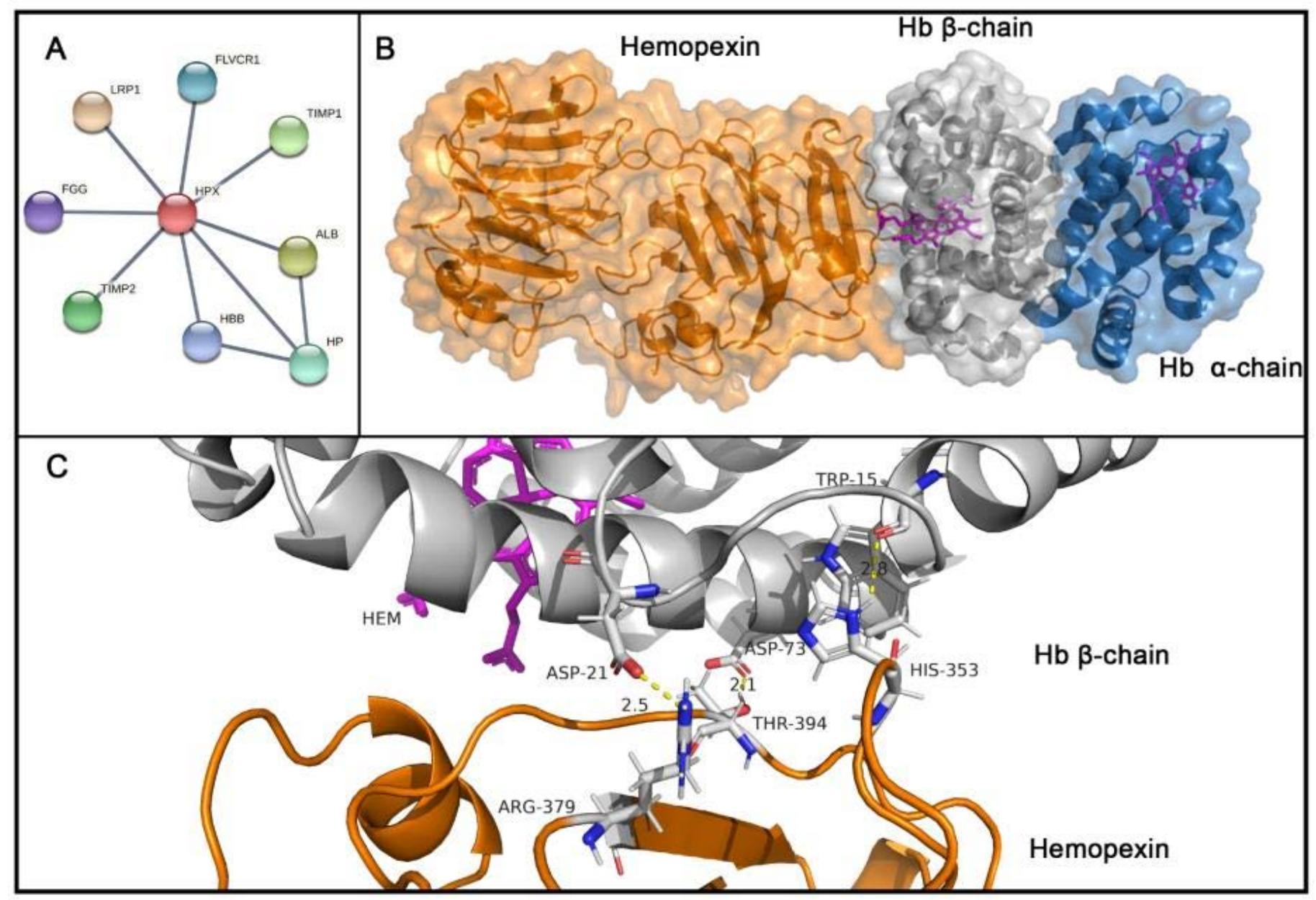

Figure 4: Hemopexon interaction with hemoglobin $(\mathrm{Hb}) \beta$-chain. (A) Network analysis of hemopexin interactome, Hx interact with albumin (ALB), haptoglobin (HP) and the $\beta$-chain of hemoglobin (HBB), fibrinogen gamma chain (FGG), metalloproteinase inhibitor 1 and 2 (TIMP1 and TIMP2), the receptor low-density lipoprotein receptor-related protein 1 (LRP1) and Feline leukemia virus subgroup C receptor-related protein 1 (FLVCR1). (B) Molecular docking of hemopexin (orange) with human hemoglobin $\beta$-chain (gray) and $\alpha$-chain (blue) (PDB 4n8t). (C) Interaction of hemoglobin $\beta$-chain (gray) with Hx (orange), representation of hotspot amino acid residues interacting by hydrogen bonds (gray sticks and yellow dashes).

\section{Discussion}

Understanding hemopexin binding motifs with heme and interactions with other proteins are crucial for its function, implications in physiology and pathology and potentially open new avenues for several disease treatment and diagnostics. The binding of mammalian hemopexin with heme was studied by X-ray crystallography, and the 3D structure of the rabbit hemopexin-heme complex revealed similar $\mathrm{N}$ and C-terminal 
domains having 4-bladed $\beta$-propeller domains that are united by a hinge sequence [14]. Heme is shown to bind to Hx by coordination of His residues (H236 and H293) in the linker region [14]. Interestingly, H293 was present in the H3 (GWHSWPIAHQ) peptide sequence with high hemin binding capacity (Signal of $~ 50 \%$ ), H236 also interacted with hemin in B22-B24 sequences (DYFMPCPGRGHGHRN, CPGRGHGHRNGTGHG, and HGHRNGTGHGNSTHH), but with lower affinity, with signal ranging from 27.5 to 37 \%. Additionally, $\mathrm{Hx}$-heme interactions were revisited by a computational and experimental study that showed histidine residues sites that can bind heme [10]. Similarly, our data demonstrated that hemin binds with residues in $\mathrm{Hx}$ binding pocket $\mathrm{H} 236 / \mathrm{H} 293$. All peptide sequences that interacted with hemin had histidine residues; notably, H373 was present in the peptides with the highest signal spots $(>90 \%)$. Also, works suggest binding heme axially between H105 and H150 [10, 13], but our data demonstrated that only H105 interacted with hemin. Many molecular interactions can mediate heme coordination, which can occur by hydrogen bonds via heme propionate groups, $\pi-\pi$ stacking between rings, electrostatic and hydrophobic interactions. The most relevant heme-coordinating amino acids are histidine, cysteine, tyrosine, methionine, and lysine at a lower frequency $[15,16]$. Identified $\mathrm{Hx}$ peptide sequence that binds hemin can be potentially used for diagnostics. There is a gap in reliable diagnostic tests for determining levels of free heme in biological samples. The severity of hemolysis and heme toxicity is currently indirectly estimated by $\mathrm{Hp}$ and $\mathrm{Hx}$ levels [11,17]. Thus, a practical and specific test is urgently needed to determine labile heme in biological fluids and tissues. Also, Hx identified peptides sequences that could be used in platforms such as nanoparticles [18] for heme scavenging therapy to improve clinical outcomes of hemolytic diseases like malaria, sickle cell disease (SCD) thalassemia, and hemorrhagic conditions. This vectorization could direct these specific heme-binding peptides to cellular compartments. The scavenging of labile heme by Hx blocked heme-driven tumor growth and metastases in a model of prostate cancer $[19,20]$. Recently, the association of $\mathrm{Hb}$ and heme levels with $\mathrm{AD}$ [9] could also bring new applications for heme-binding protein to prevent this neurodegenerative disease. In addition, $\mathrm{Hb}$ and heme are implicated in a murine model of severe sepsis [21]. Another application of hemopexin is to prevent acute adverse effects in blood transfusions after prolonged storage of red blood cells (RBC); transfusion of prolonged packed RBC can increase morbidity and mortality in trauma-induced hemorrhage, mainly by alterations in RBC and hemolysis [22]. Hx can be administered intravenously but have potential side effects caused by its structure and protease activity [23-25]. Thus, it is crucial to map only heme-binding sites in the Hx structure, to abolish potential risky side effects. Hemoglobin is the primary source of heme in our blood. To investigate the possible interaction between $\mathrm{Hx}$ and $\mathrm{Hb}$, analyzed a database, and the interactome showed binding to $\beta$-chain of $\mathrm{Hb}$ and other plasmatic proteins such as albumin and haptoglobin. Previously data demonstrated that $\mathrm{Hx}$ removes heme from $\mathrm{Hb}$ generating protein aggregates [26]. There is scarce data on protein-protein interactions (PPI) between $\mathrm{Hb}$ and $\mathrm{Hx}$. An analysis of interaction regions showed that five peptide sequences bind strongly to human hemoglobin chains. 
Haptoglobin binds $\mathrm{Hb}$ with high affinity, but when $\mathrm{Hp}$ is absent, $\mathrm{Hx}$ can potentiate $\mathrm{Hb}$ neurotoxicity by a pro-oxidant iron-dependent mechanism and also precipitate globin chains [27]. Additionally, another work pointed to a possible heme sequestration mechanism of hemopexin, and the presence strongly suppressed the synergistic inflammation effect of HMGB1, a nuclear and cytosolic DNA-binding protein released from damaged cells with hemoglobin and impaired cytokine release [28].

\section{Materials and Methods}

\subsection{Spot-Synthesis}

Uniprot retrieved the sequence of hemopexin (P02790). A library of 15 amino acid peptides with a 10-amino acid overlap was designed to represent the entire coding region (aa 1-462) of hemopexin and synthesized onto cellulose membranes using an Auto-Spot Robot ASP222 (Intavis, Koeln, Germany) [29, 30]. The membranes containing the synthetic peptides were washed with TBS-T and then blocked with TBS-T containing $1.5 \%$ BSA under agitation for $2 \mathrm{~h}$ at room temperature. After extensive washing with TBS-T (Trisbuffer saline, $0.1 \%$ Tween 20, pH 7.0), membranes were incubated overnight with $\mathrm{Hb}$ (5 $\mu \mathrm{g} / \mathrm{mL})$ dissolved in TBST + BSA $(0.75 \%)$ or hemin $(10 \mu \mathrm{M})$ for 2 hours. After incubation, membranes incubated with hemin were washed with TBS three times for 5 minutes, and then hemin-peroxidase-like activity was revealed using chemiluminescent substrate Super Signal R West Pico. Membranes incubated with hemoglobin were washed with TBS$\mathrm{T}$, followed by additional incubation with anti-human $\mathrm{Hb}$ antibody for $90 \mathrm{~min}$. Subsequently, the membrane was washed with TBS-T and incubated for 90 min with anti-rabbit IgG antibody conjugated to alkaline phosphatase (Sigma-Aldrich), diluted 1:5.000 in TBS$\mathrm{T}$ solution containing $0.75 \%$ BSA. Washes were performed with TBS-T followed by the substrate for chemiluminescent alkaline phosphatase Tropix ${ }^{\circledR}$ was added. Next, the membrane was washed three times with TBS-T, and then the buffer was exchanged to CBS (50 $\mathrm{mM}$ citrate-buffer saline) before adding the chemiluminescent enhancer Nitro-Block II. Chemiluminescent signals were detected on an Odyssey FC (LI-COR Bioscience) using the same conditions described previously [29] with minor modifications. Briefly, a digital image file was generated at a resolution of $5 \mathrm{MP}$, and the signal intensities were quantified using the TotalLab TL100 (v2009, Nonlinear Dynamics, Newcastle-Upon-Tyne, U.K.) software [28]. Spots with signal intensity greater than or equal to $50 \%$ of the highest signal value were considered to identify possible binding motifs.

\subsection{In silico analysis}

The peptides were inserted in a webserver (https://web.expasy.org/protparam/; Accessed on February 21, 2022). Analysis and graphical representation were created using $\mathrm{R}$ software version 4.1.1 and RStudio. Hemopexin interaction network was performed on platform STRING (https://string-db.org/; Accessed on February 21, 2022). The network nodes are proteins, and the edges represent the predicted functional associations of high confidence interactions. Protein 3D structures were visualized and analyzed using PyMOL Molecular Graphics System, Version 2.0 Schrödinger, LLC.

\subsection{Molecular docking}


This assay was performed with a human hemopexin model, using Swiss-model (https://swissmodel.expasy.org/) (PDB code - 1qjs), accessed on February 10, 2022. Protein-protein interaction was accessed using RosettaDock server (http://rosettadock.graylab.jhu.edu; Accessed on February 15, 2022) with human hemopexin and hemoglobin beta chain (PDB: 4n8t) and evaluated using the database (https://mitchell web.ornl.gov/ KF C Server/; Accessed on February 20, 2022) for Hotspot amino acid residues prediction.

\section{Conclusions}

These new insights may collectively contribute valuable information on Hx structure, motifs, and protein-protein interactions with $\mathrm{Hb}$. Binding sequences can be applied for therapeutic purposes and diagnostics. Hemopexin is under investigation to treat different diseases, mainly hemolytic diseases such as sickle cell disease, $\beta$-thalassemia, and hemorrhagic conditions. Also, has been considered to neurological and parasitic infections such as severe malaria. Our findings may interest therapeutic use as hemopexin peptides can specifically bind and scavenge heme without potential side effects. Also, hemolysis monitoring is crucial for sepsis and hemolytic diseases. Thus, a diagnostic test can be developed using these particular sequences.

Supplementary Materials: The following are available online at www.mdpi.com/xxx/s1, Table S1: Table S1: Linear mapping of hemopexin interaction with hemin and hemoglobin in the SPOT synthesis array (15 residues with overlapping of 10). The signal intensity of interaction regions of hemopexin sequences. Each peptide was identified by the Spot-synthesis membrane position numbering. Spot intensities below 50\% were considered as background. Figure S1: Figure S1: Interaction between hemopexin with hemoglobin. The server performed the simulation (https://rosie.graylab.jhu.edu/docking2/), generating 1000 structures represented by points in the graph. The ten structures with the best score in the test in order of classification by energy are described in dark blue. Table S2: Prediction of Hot spot interactions between Hemopexin and Hemoglobin $\beta$-chain using bioinformatics (https://mitchell-web.ornl.gov/KFC_Server/).

Author Contributions: Conceptualization, G.C.L., and S.G.D-S.; methodology, G.C.L., P.N.P.; software, G.C.L.; formal analysis, S.G.D-S., G.C.L. and P.N.P.; data curation, G.C.L., and S.G.D-S..; writing-original draft preparation, G.C.L.; writing - review and editing, G.C. and S.G.D-S; supervision, S.G.D-S.; project administration, G.C.L., S.G.D-S.; funding acquisition, C.M.M., and S.G.D-S. All authors have read and agreed to the published version of the manuscript.

Funding: This research was funded by Carlos Chagas Filho Foundation for Research Support of the State of Rio de Janeiro/FAPERJ (grant number \#110.198-13) and the Brazilian Council for Scientific Research (CNPq grant number 467.488.2014-2 and 301744/2019-0). Funding was also provided by FAPERJ (\#210.003/2018) through the National Institutes of Science and Technology Program (INCT) to Carlos Morel (INCT-IDPN).

Institutional Review Board Statement: Not applicable.

Informed Consent Statement: Not applicable.

Data Availability Statement: The data presented in this study are available in Supplemental material and on request from the corresponding author.

Acknowledgments: G.C.L. and P.N.P are Post-Doc fellows from CNPq and CAPES/CDTS, respectively.

Conflicts of Interest: The authors declare no conflict of interest. 


\section{References}

1. Tolosano, E.; Altruda, F. Hemopexin: structure, function, and regulation. DNA Cell Biol. 2002; 21: 297-306. doi:10.1089/ 104454902753759717

2. Delanghe, J.R.; Langlois, M.R. Hemopexin: a review of biological aspects and the role in laboratory medicine. Clin Chim Acta 2001; 312: 13-23. doi:10.1016/s0009-8981(01)00586-1

3. Tolosano, E.; Fagoonee, S.; Morello, N.; Vinchi, F.; Fiorito, V. Heme scavenging and the other facets of hemopexin. Antioxid Redox Signal. 2010; 12: 305-320. doi:10.1089/ars.2009.2787

4. Kumar, S.; Bandyopadhyay, U. Free heme toxicity and its detoxification systems in human. Toxicol Lett 2005; 157: 175-88. doi: 10.1016/j.toxlet.2005.03.004,

5. Eldor, A.; Rachmilewitz, E.A. The hypercoagulable state in thalassemia. Blood. 2002; 99: 36-43. doi:10.1182/ blood. v99.1.36

6. Chiabrando, D.; Vinchi, F.; Fiorito, V.; Mercurio, S.; Tolosano, E. Heme in pathophysiology: a matter of scavenging, metabolism, and trafficking across cell membranes. Front Pharmacol. 2014; 5: 61. doi:10.3389/fphar.2014. 00061

7. Hahl, P.; Davis, T.; Washburn, C.; Rogers, J.T.; Smith, A. Mechanisms of neuroprotection by hemopexin: modeling the control of heme and iron homeostasis in brain neurons in inflammatory states. J Neurochem 2013; 125: 89-101. doi:10.1111/jnc.12165

8. Vinchi, F.; Costa da Silva, M.; Ingoglia, G.; Petrillo, S.; Brinkman, N.; Zuercher, A.; Cerwenka, A.; Tolosano, E.; Muckenthaler, M.U. Hemopexin therapy reverts heme-induced proinflammatory phenotypic switching of macrophages in a mouse model of sickle cell disease. Blood. 2016; 127: 473-486. doi:10.1182/ blood-2015-08-663245

9. Ashraf, A.; Ashton, N.J.; Chatterjee, P.; Goozee, K.; Shen, K.; Fripp, J.; Ames, D.; Rowe, C.; Masters, C.L.; Villemagne, V.; Hye, A.; Martins, R.N.; So, P.W. Plasma transferrin and hemopexin are associated with altered A $\beta$ uptake and cognitive decline in Alzheimer's disease pathology. Alzheimer's Res Ther 2020; 12: 72. doi:10.118 6/s13195-020-00634-1

10. Detzel, M.S.; Schmalohr, B.F.; Steinbock, F.; Hopp, M.T.; Ramoji, A.; Paul George, A.A.; Neugebauer, U.; Imhof, D. Revisiting the interaction of heme with hemopexin. Biol Chem. 2021; 402: 675-691. doi:10.1515/hsz-20 20-0347

11. Immenschuh, S.; Vijayan, V.; Janciauskiene, S.; Gueler, F. Heme as a target for therapeutic interventions. Front Pharmacol. 2017; 8: 146. doi:10.3389/fphar.2017.00146

12. Belcher, J.D.; Chen, C.; Nguyen, J.; Abdulla, F.; Zhang, P.; Nguyen, H.; Nguyen, P.; Killeen, T.; Miescher, S.M.; Brinkman, N.; Nath, K.A.; Steer, C.J.; Vercellotti, G.M. Haptoglobin and hemopexin inhibit vaso-occlusion and inflammation in murine sickle cell disease: Role of heme oxygenase-1 induction. PLoS One 2018; 13 :e0196455. doi:10.1371/journal.pone.0196455

13. Karnaukhova, E.; Owczarek, C.; Schmidt, P.; Schaer, D.J.; Buehlerm, P.W. Human plasma and recombinant hemopexins: Heme binding revisited. Int J Mol Sci. 2021; 22: 1199. doi: 10.3390/ijms22031199.

14. Paoli, M.; Anderson, B.F.; Baker, H.M.; Morgan, W.T.; Smith, A.; Baker, E.N. Crystal structure of hemopexin reveals a novel high-affinity heme site formed between two beta-propeller domains. Nat Struct Biol. 1999;6:926-931. doi: 10.1038/13294

15. Li, T.; Bonkovsky, H.L.; Guo, J. Structural analysis of heme proteins: implications for design and prediction. BMC Struct Biol 2011; 11: 13. doi: 10.1186/1472-6807-11-13.

16. Wißbrock, A.; George, A.A.P.; Brewitz, H.H.; Kühl, T.; Imhof, D. The molecular basis of transient heme-protein interactions: Analysis, concept, and implementation. Biosci Rep 2019; 39: BSR20181940. doi: 10.1042/BSR20181940

17. Ruiz EF, Cervantes MA. Diagnostic approach to hemolytic anemias in the adult. Rev Bras Hematol Hemoter 2015; 37: 423-425. doi:10.1016/j.bjhh.2015.08.008

18. Jeong, W.J.; Bu, J.; Kubiatowicz, L.J.; Chen, S.S.; Kim, Y.; Hong, S. Peptide-nanoparticle conjugates: a next generation of diagnostic and therapeutic platforms? Nano Converg 2018; 5: 38. doi:10.1186/s40580-018-0170-1

19. Canesin, G.; Di Ruscio, A.; Li, M.; Ummarino, S.; Hedblom, A.; Choudhury, R.; Krzyzanowska, A.; Csizmadia, E.; Palominos, M.; Stiehm, A.; Ebralidze, A.; Chen, S.Y.; Bassal, M.A.; Zhao, P.; Tolosano, E.; Hurley, L.; Bjartell, A, Tenen DG, Wegiel B. Scavenging of labile heme by hemopexin is a key checkpoint in cancer growth and metastases. Cell Rep 2020; 32: 108181. doi: 10.1016/j.celrep.2020.108181 
20. Fiorito, V.; Tolosano, E. Hemopexin and cancer. Int J Mol Sci. 2022; 23: 997. doi: 10.3390/ijms23020997

21. Larsen, R.; Gozzelino, R.; Jeney, V.; Tokaji, L.; Bozza, F.A.; Japiassú, A.M.; Bonaparte, D.; Cavalcante, M.M.; Chora, Â.; Ferreira, A.; Marguti, I.; Cardoso, S.; Sepúlveda, N.; Smith, A.; Soares, M.P. A central role for free heme in the pathogenesis of severe sepsis. Sci Transl Med. 2010; 2: 51ra71. doi:10.1126/scitranslmed.3001118

22. Graw, J.A.; Mayeur, C.; Rosales, I.; Liu, Y.; Sabbisetti, V.S.; Riley, F.E.; Rechester, O.; Malhotra, R.; Warren, H.S.; Colvin, R.B.; Bonventre, J.V.; Bloch, D.B.; Zapol, W.M. Haptoglobin or hemopexin therapy prevents acute adverse effects of resuscitation after prolonged storage of red cells. Circulation 2016; 134: 945-960. doi:10.1161/CIRCULATIONAHA .115.019955

23. Pukajło-Marczyk, A.; Zwolińska, D. Involvement of hemopexin in the pathogenesis of proteinuria in children with idiopathic nephrotic syndrome. J Clin Med 2021; 10: 3160. doi:10.3390/jcm10143160

24. Hernández, C.; Garcia-Ramírez, M.; Simó, R. Overexpression of hemopexin in the diabetic eye: a new pathogenic candidate for diabetic macular edema. Diabetes Care 2013; $36: 2815-2821$. doi:10.2337/dc12-2634

25. Spiller, F.; Costa, C.; Souto, F.O.; Vinchi, F.; Mestriner, F.L.; Laure, H.J.; Alves-Filho, J.C.; Freitas, A.; Rosa, J.C.; Ferreira, S.H.; Altruda, F.; Hirsch, E.; Greene, L.J.; Tolosano, E.; Cunha, F.Q. Inhibition of neutrophil migration by hemopexin leads to increased mortality due to sepsis in mice. Am J Respir Crit Care Med 2011; 183, 922-931. doi: 10.1164/rccm.201002-0223OC

26. Hrkal, Z.; Vodrázka, Z.; Kalousek, I. Transfer of heme from ferrihemoglobin and ferrihemoglobin isolated chains to hemopexin. Eur J Biochem 1974; 43: 73-78. doi:10.1111/j.1432-1033.1974.tb03386.x

27. Chen-Roetling, J.; Ma, S.K.; Cao, Y.; Shah, A.; Regan, R.F. Hemopexin increases the neurotoxicity of hemoglobin when haptoglobin is absent. J Neurochem 2018; 145: 464-473. doi:10.1111/jnc.14328

28. Lin, T.; Sammy, F.; Yang, H.; Thundivalappil, S.; Hellman, J.; Tracey, K.J.; Warren, H.S. Identification of hem opexin as an antiinflammatory factor that inhibits synergy of hemoglobin with HMGB1 in sterile and infectious inflammation. J Immunol. 2012; 189: 2017-2022. doi:10.4049/jimmunol.1103623

29. De-Simone, S.G.; Gomes, L.R.; Napoleão-Pêgo, P.; Lechuga, G.C.; Pina, J.C.; Silva FR. Identification of linear B epitopes liable for the protective immunity of diphtheria toxin. Vaccines 2021; 9: 313. https://doi.org/10.3390/vaccines9040313

30. Lechuga, G.C.; Souza-Silva, F.; Sacramento, C.Q.; Trugilho, M.R.O.; Valente, R.H.; Napoleão-Pêgo, P.; Dias, S.S.G.; FintelmanRodrigues, N.; Temerozo, J.R.; Carels, N.; Alves, C.R.; Pereira, M.C.S.; Provance, D.W. Jr.; Souza, T.M.L.; De-Simone, S.G. SARS-CoV-2 proteins bind to hemoglobin and its metabolites. Int J Mol Sci. 2021; 22: 9035. doi:10.3390/ijms22169035 